\title{
Post-mortem dissection of COVID-19: a pathogenic role for macrophages?
}

\author{
Prasad P. Velu ${ }^{1,2}$, Christopher D. Lucas ${ }^{1,2,3^{*}}$ (D) and Andrew Conway Morris ${ }^{4,5^{*}}$ (D)
}

(C) 2021 Springer-Verlag GmbH Germany, part of Springer Nature

Acute respiratory failure is the leading cause of death in severe coronavirus disease 2019 (COVID-19). By combining rapid tissue sampling at autopsy with high dimensional analyses, including measurement of immune cells, proteins and RNA, unparalleled insights into the mechanisms of dysregulated inflammation in COVID-19 have been obtained [1-4]. Here we summarise some of the conceptual advances revealed by post-mortem studies of severe COVID-19, and make particular reference to the apparent dominance of macrophages, how this may relate to disease pathophysiology, and the opportunities for targeted therapeutic intervention.

\section{Pulmonary immunopathology drives disease severity in COVID-19}

Within the lung direct viral cytotoxicity is most notable in epithelial cells; however, the dominant pathophysiological mechanism driving severe disease appears to be virus-triggered immunopathology. The temporal and spatial disconnect between viral detection and pulmonary damage-where presence of viral RNA and protein is not always correlated with a local or proportional immune response and inflammation occurs in areas devoid of viral presence-coupled with the mortality benefits of anti-inflammatory therapies (corticosteroids and Interleukin 6 (IL-6) blockade), argues strongly for this [5-7].

The insights gained from multi-modal cellular analysis implicate specific cell types and pathways driving pulmonary immunopathology, pointing to potential therapeutic

\footnotetext{
*Correspondence: christopher.lucas@ed.ac.uk; ac926@cam.ed.ac.uk ${ }^{1}$ Centre for Inflammation Research, Edinburgh BioQuarter, Queen's Medical Research Institute, University of Edinburgh, Edinburgh, UK ${ }^{4}$ Division of Anaesthesia, Department of Medicine, Level 4, Addenbrooke's Hospital, University of Cambridge, Hills Road, Cambridge CB2 OQQ, UK

Full author information is available at the end of the article
}

options. Histologically, diffuse alveolar damage (DAD) is the common, predominant pattern identified in COVID19, Influenza H1N1 and SARS; however, microthrombi occur more frequently in COVID-19 compared with H1N1 (57\%, 24\%) [8]. Within COVID-19 lung tissue, monocyte-macrophage lineage cells appear particularly dominant and are greatly expanded in number even when compared to viral acute respiratory distress syndrome arising from influenza [3, 4]. In contrast to bacterial pneumonia, where neutrophils predominate and macrophage expansion is less marked [3, 4], COVID-19 lungs show lower absolute numbers of neutrophils, especially in severe infection and later stages of disease (Fig. 1). [3, 4]. Furthermore, the expanded COVID-19 macrophages are most frequently seen in extravascular lung tissue and are predominantly 'interstitial' rather than 'alveolar' in nature, suggesting that recruitment of monocyte-macrophage lineage cells from extrapulmonary sites is promoting macrophage expansion within lung tissue $[1,3]$. In keeping with this, high genotype-inferred pulmonary expression of the monocyte-macrophage chemotactic receptor CCR2 is associated with severe disease, and numerous monocyte/macrophage chemotactic factors are elevated in blood, bronchoalveolar lavage fluid and whole lung tissue in COVID-19 [4, 9, 10]. These expanded macrophage populations are highly activated and with aberrant expression of key inflammation-associated molecules, often alongside expression of genes associated with tissue repair and fibrogenesis [2, 11, 12]. Interestingly, while epithelial cells are clearly the main viral target of SARS-CoV-2 infection, studies have reported viral $S$ protein within macrophages $[1,3]$. Whether this represents primary infection of macrophages, or is merely a consequence of macrophage engulfment of infected epithelial cells, remains to be determined.

Pulmonary vascular and endothelial pathology are also key features of fatal COVID-19, with multiple reports of

\section{Springer}




\section{Fatal COVID-19: A hypothesis linking viral toxicity and monocyte/macrophage mediated immunopathology}

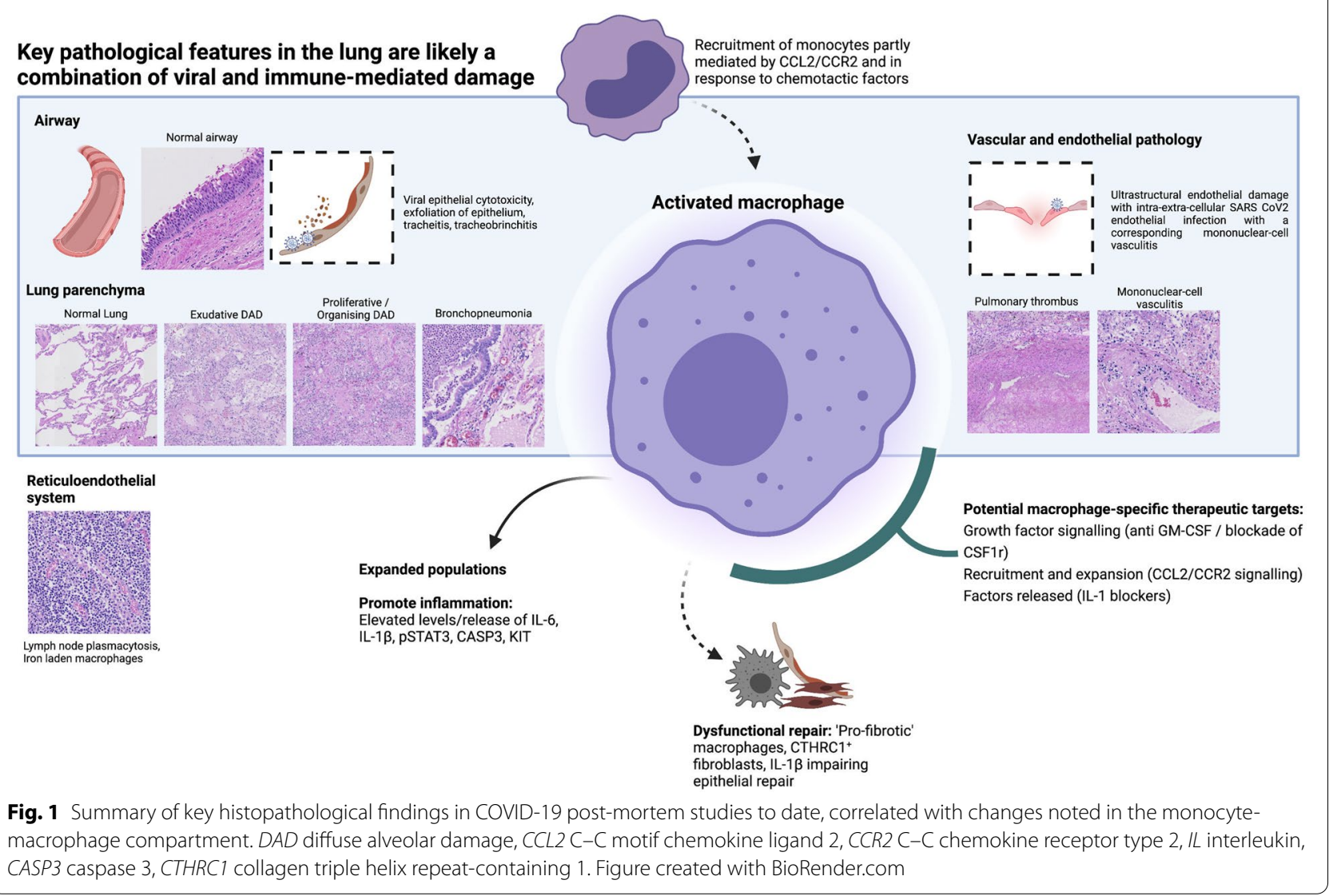

thrombotic complications occurring despite anticoagulation therapy [11]. Ultrastructural endothelial damage is prevalent with intra- and extra-cellular SARS-CoV-2 endothelial infection reported, as well as a mononuclearcell vasculitis affecting the intimal layer of small and medium-sized pulmonary arteries, mainly comprising $\mathrm{MRP}^{+}$monocyte-macrophage cells (and not $\mathrm{T}$ cells as reported in influenza) (Fig. 1) [1]. These observations connect immunopathology with endothelial disruption and dysfunction and is consistent with immunothrombosis occurring in COVID-19 [1, 13]. Whether COVID-19 related endothelial dysfunction and thrombi can be targeted by anti-inflammatory strategies awaits clarification.

\section{Dysregulated repair driven by macrophages in the lung microenvironment}

Given the key roles played by macrophages in regulating tissue homeostasis and repair, the non-inflammatory downstream effects of aberrant macrophage activation in COVID-19 have also been investigated. Evidence of impaired lung regeneration at the level of alveolar epithelial cells is observed, with the potential for monocyte/macrophage-derived interleukin 1 beta (IL-1b) impairing epithelial repair and aiding neutrophil recruitment $[2,10]$. Reduced absolute numbers of endothelial cells, and expanded populations of mesenchymal cells (particularly collagen triple helix repeat-containing protein $1\left(\mathrm{CTHRC1}^{+}\right)$pathological fibroblasts $)$have been noted $[3,14]$. The emergence of these populations, alongside their demonstrable close proximity to macrophages (with some expressing potentially pro-reparative but also profibrotic genes such as triggering receptor expressed on myeloid cells 2 (TREM2) and sphingosine-1-phosphate phosphatase 1 SPP1), could account for the higher degrees of fibrosis-considered a feature of dysfunction repair-noted in COVID-19 lungs [3, 4].

\section{Extrapulmonary macrophage activation and mobilisation}

While most studies have focussed on circulating cell and pulmonary abnormalities, evidence also exists for extrapulmonary macrophage abnormalities. Iron 
laden bone marrow macrophages are frequent in severe COVID-19 (Fig. 1). Multiple infectious diseases are known to impact on iron metabolism [15], with proinflammatory molecules including IL-6 and IL-1beta established modulators of iron handling pathways [1, 15]. Exposure to high levels of iron also induce inflammatory cytokine release by macrophages and lymphocytes, which can set-up self-reinforcing cycles of immune-cell activation, with macrophage haemophagocytosis a prominent feature of the systemic inflammatory Macrophage Activation Syndrome. Notably, haem ingestion also significantly impairs antimicrobial functions of macrophages [15], which may influence the high rates of secondary infection noted in patients with COVID-19. The extent to which haemophagocytosis is a driver of the pathology seen in COVID-19 awaits formal testing. Extensive abnormalities in splenic tissue are also described, including increased abundance of macrophage colony stimulating factor (M-CSF/CSF-1), an important mediator of proliferation and differentiation of monocytes, macrophages and their precursors. Whether splenic monocyte-macrophages directly contribute to the increased expansion of these cells within pulmonary tissue awaits clarification.

\section{Potential therapeutic angles}

Whether targeted manipulation of monocyte/macrophage pathways will lead to patient benefits awaits clinical trials, but given the multiple studies implicating these cells as potentially causal in severe disease, this supports prioritising the testing of macrophage-targeting agents. Several approaches are possible, including macrophage ablation via interfering with growth factor signalling (e.g. anti GM-CSF or blockade of CSF1R), attenuating monocyte/macrophage recruitment and expansion (e.g. targeting CCL2/CCR2 signalling) or targeting factors liberated from highly activated pro-inflammatory macrophages (e.g. IL-1 blockers) (Fig. 1).

\section{Conclusions and future perspectives}

Although we must approach findings from post-mortem studies with caution, given that they are by definition obtained from patients who have not survived, multimodal in-vivo investigations support these findings being generalisable to patients with severe disease. The implications of the findings of post-mortem studies for intensive care clinicians are several. First, they provide key data for the identification and trialling of targeted therapeutics, and provide confidence in the trial results of proven therapies. Second, they indicate where, in the absence of COVID-19 specific trials indicating differently, we can apply knowledge from other related diseases, such as the evidence-based management of acute respiratory distress syndrome (ARDS) and sepsis. Third, the approaches taken to investigate SARS-CoV-2 infection serves as a platform to address critical knowledge gaps in the management and treatment of many different critical illnesses and illustrate the value of post-mortem examinations, encouraging restoration of the prominence of this technique to inform both clinical practice and research.

\begin{abstract}
Author details
${ }^{1}$ Centre for Inflammation Research, Edinburgh BioQuarter, Queen's Medical Research Institute, University of Edinburgh, Edinburgh, UK. ${ }^{2}$ Department of Respiratory Medicine, Royal Infirmary of Edinburgh, Edinburgh, UK. ${ }^{3}$ Institute for Regeneration and Repair, Edinburgh BioQuarter, University of Edinburgh, Edinburgh, UK. ${ }^{4}$ Division of Anaesthesia, Department of Medicine, Level 4, Addenbrooke's Hospital, University of Cambridge, Hills Road, Cambridge CB2 0QQ, UK. ${ }^{5}$ John V Farman Intensive Care Unit, Addenbrooke's Hospital, Cambridge, UK
\end{abstract}

\section{Acknowledgements}

ACM is supported by a Medical Research Council Clinician Scientist Fellowship (MR/V006118/1). CDL is supported by a Wellcome Trust Clinical Research Career Development Fellowship (206566/Z/17/Z) and receives funding from the UKRI-NIHR COVID-19 Call (MR/V028790/1). PPV is supported by a Wellcome Trust PhD Training Fellowship for Clinicians (214179/Z/18/Z). The authors thank David Dorward for assistance with image selection.

\section{Author contributions}

$\mathrm{CL}$ and $\mathrm{ACM}$ conceived the article, PPV, CL and ACM generated the outline, PPV and $C L$ wrote the first draft and generated the figure, PPV, CL and ACM critically revised the manuscript for important intellectual content, and agreed the final submitted version of the manuscript.

Funding

None.

Availability of data and material

Not applicable.

\section{Declarations}

Conflicts of interest

All authors have declared that no conflicts of interest exist.

\section{Ethical standard}

Pathology images were provided by ICECAP (Inflammation in COVID-19: Exploration of Critical Aspects of Pathogenesis), with ethical approval granted by the East of Scotland Research Ethics Service (16/ES/0084).

\section{Publisher's Note}

Springer Nature remains neutral with regard to jurisdictional claims in published maps and institutional affiliations.

Received: 12 June 2021 Accepted: 15 August 2021 Published online: 1 September 2021

References

1. Dorward DA et al (2021) Tissue-specific immunopathology in fatal COVID-19. Am J Respir Crit Care Med 203:192-201

2. Melms JC et al (2021) A molecular single-cell lung atlas of lethal COVID19. Nature $33: 15$

3. Rendeiro AF et al (2021) The spatial landscape of lung pathology during COVID-19 progression. Nature 593:564-569 
4. Liao M et al (2020) Single-cell landscape of bronchoalveolar immune cells in patients with COVID-19. Nat Med 26:842-844

5. Ryu G, Shin HW (2021) Sars-cov-2 infection of airway epithelial cells. Immune Network 21:1-16

6. Arabi YM, Chrousos GP, Meduri GU (2020) The ten reasons why corticosteroid therapy reduces mortality in severe COVID-19. Intensive Care Med 46:2067-2070

7. Snow TAC et al (2021) Tocilizumab in COVID-19: a meta-analysis, trial sequential analysis, and meta-regression of randomized-controlled trials. Intensive Care Med. https://doi.org/10.1007/s00134-021-06416-z

8. Hariri LP et al (2021) Lung histopathology in coronavirus disease 2019 as compared with severe acute respiratory sydrome and H1N1 influenza: a systematic review. Chest 159:73-84

9. Pairo-Castineira E et al (2021) Genetic mechanisms of critical illness in COVID-19. Nature 591:92-98
10. Chua RL et al (2020) COVID-19 severity correlates with airway epithelium-immune cell interactions identified by single-cell analysis. Nat Biotechnol 38:970-979

11 Dolby HW et al (2021) Histological evidence of pulmonary microthrombosis and vasculitis in life-threatening respiratory virus diseases. Open Forum Infect Dis. https://doi.org/10.1093/ofid/ofaa640

12. Yang $L$ et al (2021) An immuno-cardiac model for macrophage-mediated inflammation in COVID-19 hearts. Circ Res 129:33-46

13. Ackermann $\mathrm{M}$ et al (2020) Pulmonary vascular endothelialitis, thrombosis, and angiogenesis in Covid-19. N Engl J Med 383:120-128

14 Tsukui T et al (2020) Collagen-producing lung cell atlas identifies multiple subsets with distinct localization and relevance to fibrosis. Nat Commun. https://doi.org/10.1038/s41467-020-15647-5

15. Nairz M, Weiss G (2020) Iron in infection and immunity. Mol Aspects Med 75:100864 